Itinéraires Itinéraires

Littérature, textes, cultures

2018-1| 2018

Le manifeste à travers les arts : devenirs d'un genre indiscipliné

\title{
T68 ou la désillusion d'une génération
}

T68 or the Disillusionment of a Generation

\section{Karel Vanhaesbrouck}

\section{(2) OpenEdition}

Journals

Édition électronique

URL : http://journals.openedition.org/itineraires/4317

DOI : $10.4000 /$ itineraires.4317

ISSN : 2427-920X

Éditeur

Pléiade

Référence électronique

Karel Vanhaesbrouck, « T68 ou la désillusion d'une génération », Itinéraires [En ligne], 2018-1 | 2018, mis en ligne le 15 septembre 2018, consulté le 16 mai 2020. URL : http://journals.openedition.org/ itineraires/4317 ; DOI : https://doi.org/10.4000/itineraires.4317

Ce document a été généré automatiquement le 16 mai 2020.

\section{(c) $(1) \odot$}

Itinéraires est mis à disposition selon les termes de la licence Creative Commons Attribution - Pas d'Utilisation Commerciale - Pas de Modification 4.0 International. 


\title{
T68 ou la désillusion d'une génération
}

\author{
T68 or the Disillusionment of a Generation
}

\author{
Karel Vanhaesbrouck
}

En 1968, l'écrivain Hugo Claus, l'acteur Alex Van Royen et le critique et théoricien Carlos Tindemans publièrent le manifeste $T 68$, avec comme sous-titre L'avenir du théâtre aux Pays-Bas du sud. Publié, avec un petit clin d'œil peut-être ironique, sous la forme d'un petit livre rouge, le texte semble né d'une profonde insatisfaction face à l'état du théâtre contemporain et proteste contre l'immobilisme et la médiocrité bourgeoise que Claus, Tindemans et Van Royen observent chez leurs confrères contemporains. Ils dénoncent une trop grande dépendance des théâtres municipaux vis-à-vis d'enjeux locaux et la place congrue que les structures existantes réservent à l'expérimentation formelle. Bien que les auteurs soient les premiers à refuser à leur texte le statut de manifeste - «ce document n'est pas un manifeste ", en voilà la phrase d'ouverture - ils publient leur projet en se servant d'un style rhétorique et combatif qui ne craint pas les assertions normatives. C'est donc en tant que manifeste que je lirai ici ce texte tout à fait passionnant. Aujourd'hui encore, T68 est un document intrigant qui pose des questions stimulantes, par exemple au sujet du sens et du non-sens des théâtres nationaux et de la fonction représentative qui y est liée. Même si le titre du manifeste renvoie à l'année magique 1968, le document est tout sauf un document typique de cette génération. Dans cet article, je souhaite non seulement dégager les lignes de force de ce manifeste "malgré lui ", mais également en entreprendre une tentative d'interprétation à partir de son contexte. Ce faisant, mon objectif sera de démontrer qu'il est impossible de dissocier T68 d'un certain nombre de facteurs contextuels, en observant que ce document s'inscrit dans un réseau discursif et historiquement déterminé et dépend de la position poétique des auteurs. Par ailleurs, je soutiendrai la thèse que, dans une mesure importante, T68 était un texte hors de son temps, en porteà-faux par rapport à celui-ci, et qu'en tant que manifeste, il a en grande partie manqué de produire son effet. Or, c'est précisément à ce moment-là qu'un texte devient vraiment un manifeste, au moment où il fait remuer la surface par trop plane de l'eau. 
2 Dans T68, Van Royen, Claus et Tindemans plaident en faveur de la mise en place d'une grande compagnie de théâtre national en Flandre qui incorporerait également un laboratoire théâtral voué à la recherche et à l'expérimentation. Leur texte n'est pas seulement la présentation d'une vision, c'est aussi, littéralement, une candidature spontanée auprès du ministre de la Culture de l'époque : la compagnie à créer serait dirigée par eux-mêmes. Ils s'inscrivent explicitement en faux contre l'ancrage local des grands théâtres municipaux qui occupent à l'époque une position relativement privilégiée, mais qui sont surtout à la merci de différents enjeux locaux. La tête la première, les auteurs ciblent directement la léthargie croissante qui semble frapper leurs confrères. L'initiateur de cette entreprise était l'acteur Alex Van Royen ${ }^{1}$ (De Roeck 1988 : 19) qui, non satisfait de la qualité de la scène contemporaine du théâtre de chambre, avait quitté la Flandre. Ce circuit théâtral s'intéressait pourtant au théâtre d'avant-garde littéraire (avec une prédilection pour le théâtre de l'absurde), mais les mises en scène continuaient de produire un «théâtre traditionaliste [...] pour l'essentiel nappé d'une sauce psychologique relativement superficielle » (Ibid.: 19). Malgré le choix d'une écriture expérimentale, dans les spectacles mêmes il n'en restait, d'après Van Royen, presque plus aucune trace. Ceux-ci n'offraient par conséquent à l'acteur que fort peu, voire pas de possibilités d'épanouissement personnel, un des dadas de Van Royen, qui était à l'époque également un professeur apprécié de l'école supérieure de théâtre, le RITCS, qui venait d'être fondée. C'est cette insatisfaction qui poussa Van Royen vers Tindemans et, ensemble, ils décidèrent de demander à Hugo Claus de s'associer à leur initiative. Une vingtaine de réunions au domicile de Claus à Nukerke, voilà ce qu'il allait falloir, en tout et pour tout, aux trois hommes de théâtre pour rédiger le manifeste. Ce texte est né dans une constellation contextuelle bien particulière : en 1967, le KNS à Anvers avait perdu sa mission de "Théâtre national ". Alors que, du côté francophone, le Théâtre national de Belgique continue de nos jours de remplir ce rôle, du côté flamand, il avait été soudainement mis fin au rêve de disposer d'une compagnie néerlandophone de théâtre national.

\section{Lignes de force}

3 «Ceci n'est pas un manifeste » : c'est donc sur cette phrase que s'ouvre la plaquette. Pourtant, leur texte correspond, comme nous le verrons, à plusieurs caractéristiques attribuées à ce genre textuel : le ton normatif et par moments exalté, le désir d'en finir avec les structures existantes sclérosées (cf. l'idée de la table rase chez les avant-gardes en général), la stratégie explicitement programmatique, la conscience de soi visionnaire des auteurs. Avec leur manifeste, qui ne se revendique donc pas comme tel, ils veulent, selon leurs propres dires, combattre la sclérose du théâtre flamand pendant les années 1960 :

Nous souhaitons démontrer que l'incapacité et l'immobilisme ne sont pas forcément les conditions normales pour faire du théâtre. Nous voulons informer le monde qu'il existe des hommes qui ont des opinions et des projets pour fonder et développer dans notre pays cette compagnie de théâtre à laquelle les Flamands ont droit et à laquelle ils aspirent. Nous voulons donner un démenti aux bureaucrates et aux hommes politiques, qui tirent profit d'un théâtre bourgeois docile et sclérosé, et leur rappeler leur devoir. Le théâtre que nous visons est un projet clair qui peut démarrer en $1968^{2}$. 
4 Avec la fin du théâtre national, les grandes compagnies de théâtre - le KNS à Anvers, le KVS à Bruxelles, le NTG à Gand - semblent avoir dégénéré en un imbroglio politique dominé par des intérêts locaux. Les ressources, l'infrastructure et les compétences sont mobilisées de manière dispersée, avec pour conséquence une anémie artistique poussée: «chacune à sa façon, nos compagnies de théâtre officielles alignent leur politique de théâtre sur le plus grand dénominateur commun ». Les directeurs de théâtre et les metteurs en scène recherchent surtout un public "approuvant leurs spectacles d'un signe de tête ", fixent des choix en évitant les risques et montent leurs spectacles systématiquement "pour le même public assoupi [qui] subit chaque représentation sans effectuer une sélection personnelle ». Le théâtre de répertoire est présenté suivant une approche archéologique, apparemment objectivante, dénuée de toute personnalité : «il manque à notre théâtre actuel un style, une volonté formelle explicite, un filigrane, une âme ». De plus, ce théâtre a perdu tout contact avec la réalité et n'est pas adapté aux problèmes et aux défis de l'époque. Il faut donc lancer une nouvelle initiative, comme l'affirment les auteurs dans un passage éminemment programmatique :

Nous faisons des suggestions basées sur cette réalité observable pour tout le monde et nous voulons changer cette situation de fond en comble. Cette nouvelle réalité s'appelle T68 [...] Nous voulons que cela change. Que les propositions de T68 deviennent réalité.

5 Ce fragment est suivi d'une phrase emblématique du genre du manifeste, plaidant en faveur d'une table rase générale: «T68 vise plus loin que le pur et simple assainissement des défigurations existantes et historiquement intouchables; il entend remettre les compteurs à zéro. » Ensuite, les auteurs s'en prennent au goût bourgeois, avec sa préférence pour un réalisme sans danger et pour des spectacles historiques insipides. "Nous en finissons avec le théâtre de papa, le plaisir bourgeois, le divertissement, le théâtre de chaise-bascule qui propose une sublimation de tous les rêves. " Tout au long de leur manifeste, les auteurs soulignent l'autonomie du théâtre en tant que forme artistique. Le théâtre, disent-ils, doit s'affranchir de messages idéologiques ${ }^{3}$ mais aussi de l'hégémonie du texte. Il doit partir à la recherche d'une «expérience maximale où le décor et le costume, la musique, le texte, l'appareil linguistique et tous les moyens scéniques se stimulent mutuellement pour créer un acte de l'imagination ". Pour cette raison, il s'agit non seulement de créer une nouvelle compagnie nationale, mais encore $d$ 'y associer un laboratoire, permettant la réalisation d'expérimentations qui doivent toujours se faire en fonction d'un spectacle définitif ${ }^{4}$. Ils prennent explicitement du recul par rapport à l'interprétation psychologique du jeu de l'acteur (Stanislawski, method acting). Le théâtre doit chercher ses propres moyens et codes représentationnels, loin $d u$ réalisme, mais également loin $d u$ théâtre psychologique rituel. L'acteur doit avoir plus d'une corde à son arc, de façon à pouvoir développer une forme d'expression spécifique à chaque pièce : l'entraînement physique et vocal fait dès lors partie intégrante du travail de laboratoire quotidien ${ }^{5}$. « T68 lancera une nouvelle tradition », lit-on dans le manifeste. Le théâtre fonctionne suivant un système de représentation autonome (ce n'est pas un hasard si Carlos Tindemans deviendra ultérieurement le premier professeur en études théâtrales en Flandre). C'est donc une forme autonome de production du sens. En puisant dans ses propres ressources, le théâtre doit être adapté au monde contemporain :

[...] nous voulons un théâtre d'aujourd'hui au milieu de personnes d'aujourd'hui [...]

À travers son homogénéité et les prestations de sa compagnie, T68 veut s'adresser 
aux milliers de personnes qui tournent actuellement le dos au théâtre parce qu'elles n'y trouvent que de l'auto-complaisance bourgeoise ou des expérimentations obscures et tièdes.

6 Van Royen, Claus et Tindemans cherchent clairement à renouer avec l'ancien rêve du Vlaamse Volkstoneel qui avait bénéficié d'une popularité sans précédent pendant l'entre-deux-guerres tout en présentant un langage dramaturgique expérimental à un grand public. À travers cette vision large et populaire du théâtre, T68 entend proposer un théâtre de répertoire qui se focalise sur l'événement scénique (plutôt que sur l'objet littéraire même). Chaque texte est le point de départ d'un événement théâtral qui veut remettre en question l'époque contemporaine. L'adaptation de classiques existants fait partie de l'ADN de T68: « la seule condition est que ces textes stimulent l'imagination, transmettent une sensation véritable et produisent un spectacle a-réaliste ». Claus, Van Royen et Tindemans veulent rendre au théâtre son rôle sociétal : T68 doit pousser le spectateur à tourner le dos à son écran de télévision et à son faux réalisme.

7 À la fin du manifeste, le ton devient très pragmatique. Les trois auteurs n'hésitent pas à traduire leur projet en termes budgétaires. Le calcul est formulé en exactement quinze lignes, non dénuées d'ironie :

Nous avons pris en compte un montant global de dépenses d'environ 7 millions de francs, nous escomptons une recette d'un million de francs, ce qui porte le montant à subsidier à 6 millions de francs. Tout observateur neutre réalise que ce montant est le minimum absolu. Il convient dès lors d'observer une minute de silence.

\section{Révolutionnaire ou réactionnaire?}

Même si les auteurs refusent de coller l'étiquette de manifeste sur leur texte, ils ne le considèrent pas moins comme révolutionnaire, ainsi que l'affirme Carlos Tindemans dans une interview où il jette un regard rétrospectif sur l'aventure. «Le mot révolutionnaire est évidemment un peu facile, mais, ce que nous proposions s'écartait tellement des dispositions habituelles que nous pensions qu'il serait impossible de gagner les décideurs politiques à notre cause » (De Roeck 1988: 19). C'est ce qui s'est produit. Leur proposition n'a pas été capable de combattre la politique régionaliste fondée sur le principe "Divisez et régnez ", qui définissait à cette époque la politique culturelle en Flandre. Constatant que l'impact du manifeste restait limité, Claus a décidé de réaliser un tirage de la brochure à compte d'auteur chez l'éditeur Sanderus à Audenaerde. Tirée à deux cents exemplaires, la brochure fut diffusée auprès de la presse écrite, radiophonique et télévisée aux Pays-Bas et en Flandre mais ne fit pas couler beaucoup d'encre. $T 68$ était condamné à rester lettre morte. La réaction standard était une apathie généralisée. Dans la brochure diffusée par les soins de Claus, on pouvait lire à la première page : «Nous avons été informés que cette proposition a été mise au frigo pour une période indéterminée. » Alfons Van Impe, un fonctionnaire culturel, affilié au CVP, du ministère de la Culture néerlandaise, était tout sauf un partisan des propositions du manifeste, c'est le moins que l'on puisse dire. Dans un article (Streven, le mois d'avril 1969), Van Impe avait critiqué le projet T68. Tindemans reprend, avec une légère touche d'ironie, le jugement de Van Impe, dans une de ces notices:

Reste le problème de T68. Van Impe n'est pas contre. Mais cette «profession de foi en robe de chambre » n'est qu'un «besoin dévorant de se faire valoir». Il ne peut en résulter que du «dynamisme touche-à-tout » et donc du «dilettantisme » et il 
contient un certain nombre de thèses qui iraient à l'encontre des leçons tirées de l'expérience. Par ailleurs, il renvoie également à une "vision intellectualiste et donc stérile » et T68 n'intéresserait que la couche supérieure de la population ${ }^{6}$.

L'attention limitée qu'a connue le manifeste est sans doute liée à sa position particulière dans le climat de cette époque. Non seulement le projet est par trop ambitieux et audacieux pour les décideurs politiques contemporains, mais le monde artistique, notamment la jeune génération, réagit en outre la plupart du temps de manière apathique à $T 68$, considéré tout au plus comme un projet sympathique. Alors qu'aux Pays-Bas, dans le sillage du mouvement Provo, l'Aktie Tomaat, une action lancée par des jeunes plaidant en faveur d'une reconquête du théâtre par le politique, devait se manifester un an plus tard, le texte de T68 ne visait pas le renouveau social mais le potentiel d'innovation du théâtre en tant que tel. Au cœur du manifeste se trouve la thèse décrite ultérieurement par Tindemans comme une "ré-esthétisation du théâtre »: le théâtre est une forme artistique indépendante, autonome, dotée d'un langage et d'un système de représentation propres (De Roeck $1988: 20$ ). Avec cet accent permanent sur l'autonomie, les trois auteurs se positionnent à l'écart du climat contemporain, dominé par la contestation anti-autoritaire et l'engagement politicorévolutionnaire. Dans les années 1970, le théâtre flamand traversera une évolution exactement opposée à celle préconisée par le manifeste : dans le théâtre politique de compagnies comme le Trojaanse Paard (le Cheval de Troie) et la Internationale Nieuwe Scène (Nouvelle scène internationale), la dimension esthétique est complètement subordonnée au message politique.

10 À la lumière de ces discussions, deux événements méritent qu'on y regarde de plus près : le passage en Belgique du Living Theatre en 1962 et la première de Thyestes à Bruxelles en 1966. Carlos Tindemans était un critique alerte de la première compagnie, Hugo Claus était l'auteur et le metteur en scène de Thyestes et Alex Van Royen était l'un des acteurs de la pièce. Une analyse approfondie de ces deux événements nous mènerait trop loin ici et dépasse les limites de la présente contribution, mais ils nous aideront à mieux appréhender l'inscription historique du manifeste T68, les motivations sous-jacentes et les préoccupations de ses rédacteurs. En 1962, le Living Theatre présente The Connection et The Apple, deux pièces de Jack Gelber, à Anvers et à Bruxelles. Avec leur théâtre inspiré d'Artaud, proche du happening, Malina et Back voulaient créer un impact culturel et politique immédiat sur le spectateur. Le 24 mai 1962, Tindemans écrit un compte rendu pour De Nieuwe Gazet ${ }^{7}$, et comprend que ces artistes font du théâtre qui renoue avec une autre essence : « dans le tourbillon de la négation totale, tout ce que nous avons considéré indispensable jusqu'à présent est absorbé dans l'air de plus en plus léger : une pièce bien ficelée, un spectacle clos, une salle passive, une action limitée » (Crombez 2014 : 77).

11 L'œuvre du Living Theatre, comme le comprend Tindemans dès 1962, vise une présence performative, au-delà de toute forme de représentation théatrale. Ce théâtre veut, tout comme le happening, être en premier lieu un "événement». Cinq ans plus tard, la même idée apparaîtra dans le manifeste $T 68$ : «si nous pouvons réactiver le théâtre et lui restituer l'appel d'une expérience, d'une sensation et d'une fête ». Dans son compte rendu pour De Nieuwe Gazet, Tindemans souligne en permanence la rupture avec la langue théâtrale de l'époque. Il se saisit explicitement du Living Theatre comme d'un levier pour «indiquer au paysage du théâtre flamand anno 1962 ses nombreux manquements et responsabilités manquées" (Crombez 2014: 82). Une analyse 
exactement identique à celle qui, cinq ans plus tard, allait être à l'origine du manifeste T68.

Environ deux ans avant la publication du manifeste eut lieu, au Palais des beaux-arts de Bruxelles, la première d'un autre spectacle: Thyestes de Hugo Claus par la compagnie Toneel Vandaag, interprété, entre autres, par Rudi Van Vlaenderen, Ton Lutz et Alex Van Royen. Cette pièce annonçait déjà les recherches formelles radicales et les idées sur le théâtre autonome qui allaient être défendues ultérieurement avec bravoure par le manifeste. La production, considérée encore aujourd'hui comme une césure importante dans l'histoire du théâtre flamand de l'après-guerre, se situait complètement à l'écart du langage théâtral habituel, tant au centre du système théâtral en Belgique (l'écriture scénique de Claus était par trop radicale) que dans la périphérie de ce système (Claus était resté éloigné de toute une série de phénomènes extra-théâtraux comme le happening).

La découverte d'Artaud et de ses conceptions sur le théâtre de la cruauté a joué un rôle crucial dans le développement des idées de Claus. Grotowski qui, dans sa recherche de nouvelles formes d'expression théâtrale, a tenté de traduire Artaud vers un programme rigoureusement physique, est une autre figure influente de cette époque. Toutes ces conceptions, comme celles de T68, ont en commun de placer l'acteur au centre de l'attention : "par l'intermédiaire d'Artaud et de Grotowski, retentit l'appel d'un autre théâtre plus rituel, non seulement orienté vers la psychologie mais vers l'expérience physique [...], la confrontation et la provocation » (Van Den Dries $2007: 139)^{8}$. Inspiré par ces évolutions, Claus met en scène son adaptation de la pièce de Sénèque comme une confrontation avec les désirs les plus secrets que nous nourrissons. Les acteurs crient, larmoient, hurlent, soufflent et sifflent, exhibent leur corporalité exaltée, orientée vers l'expressivité. Le décor est monumental et abstrait avec, au beau milieu, une énorme tête de bélier. Louis De Meester a créé un décor sonore électronique dans lequel se succèdent des sons menaçants, des tons perçants et un chaos impénétrable. À tous les niveaux, Claus a cherché des images de nausée et de destruction-certains estimaient qu'avec le spectacle Thyestes, il avait transgressé les limites du bon goût. Il a en fait suivi le chemin radical de la recherche d'un nouveau langage formel, d'une façon d'utiliser l'artificialité du théâtre pour vraiment affecter le spectateur, au-delà de tout réalisme. Son Thyestes était une tentative de re-théâtraliser le théâtre contemporain : le texte et la mise en scène devaient contaminer le spectateur comme un virus, rendre caduque toute forme de dégustation bourgeoise d'une esthétique littéraire raffinée.

Dans son compte rendu du spectacle en 1967, Tindemans décrit l'œuvre comme « une contribution constructive à l'introduction d'une nouvelle perspective dans notre propre théâtre » (Tindemans $1967: 47$ ). Avec sa tentative de renouvellement radical de la langue théâtrale, Claus se trouvait quasiment isolé. Pour les théâtres officiels, ce spectacle était par trop radical, mais, d'un autre côté, la pièce n'était pas adaptée à l'orientation du théâtre des jeunes générations des années 1960 qui recherchaient une forme d'engagement plus radicale, plus explicitement politique. Pour les jeunes, la recherche d'un autre langage formel était d'une importance tout à fait secondaire. C'est la raison pour laquelle Thyestes n'a pas provoqué de tremblement de terre, mais bien un affaissement de terrain, comme l'affirme, à juste titre, Luk Van Den Dries: «le spectacle laissait un cratère sur lequel nous jetons aujourd'hui un regard étonné " (Van Den Dries 2007 : 142). Du point de vue de l'histoire du théâtre, le spectacle a un 
statut essentiellement ambigu. Porté par une volonté radicale de changement, il voulait être un moment de rupture, mais ne devait guère connaître de suite ni produire d'effet: un lot exactement identique sera réservé au manifeste T68. Même si les trois auteurs réalisaient parfaitement qu'à la fin des années 1960, le théatre flamand était complètement bloqué au niveau institutionnel, et même s'ils ont essayé d'injecter dans le centre politique contemporain des concepts de toute une série de projets d'avantgarde expérimentaux, leur projet est resté sans écho et sans impact. Des graines tombées sur les roches, des perles jetées aux cochons, sans plus. Parfois, un manifeste arrive au mauvais moment, il est out of place et out of time, disjoncté, hors des gonds. On a beau crier, le seul effet qu'il engendre est un haussement d'épaules généralisé. T68 se révèle ainsi être un document historiographique tout à fait paradoxal: un texte rhétorique et combatif qui nie explicitement son propre statut de manifeste, mais qui, en même temps, s'empare de toutes ses caractéristiques, comme nous venons de l'expliquer. T68 est bel et bien un manifeste qui visait un impact performatif, c'est-àdire transformer le système artistique qu'il critiquait. Ses auteurs cherchaient à multiplier son impact en le diffusant largement; ils cherchaient donc un écho à leur texte. Le statut de T68 est donc, c'est le moins qu'on puisse en dire, complexe et même contradictoire : il fait usage de l'appareil rhétorique du manifeste d'avant-garde, en se distanciant en même temps de ce genre typique de l'avant-garde historique, comme si ses auteurs prenaient déjà en compte le peu de réactions suscitées par leur texte et l'impossibilité de leur projet. Peut-être est-ce l'essence même du genre du manifeste : l'impossibilité d'un rêve, celui de faire remuer la surface de l'eau?

\section{BIBLIOGRAPHIE}

Crombez, Thomas, 2014, Arm theater in een gouden tijd. Ritueel en avant-garde na de Tweede Wereldoorlog, Tielt, Lannoo Campus.

Decreus, Freddy, 1996, « Claus regisseert Thyestes bij Toneel Vandaag », dans R. Erenstein (dir.), Een theatergeschiedenis der Nederlanden. Tien eeuwen drama en theater in Vlaanderen en Nederland, Amsterdam, Amsterdam University Press, p. 738-743.

Dries, Luk Van Den, 2007, «Thyestes: een grondverzakking in het Vlaamse theater », Documenta. Toneelstof : Route 66, vol. 25, $\mathrm{n}^{\circ}$ 2, p. 132-141.

Roeck, Jef de, 1988, « T68, een afgedreven voorstel », Etcetera, n² 21-22, p. 17-21.

Thielemans, Johan, 2007, « Schouwburgen in bloei en verval », Documenta. Toneelstof: Route 66, vol. $25, \mathrm{n}^{\circ} 2$, p. 77-94.

Tindemans, Carlos, 1967, « Claus' Thyestes op Hollandfestival. Poging tot theatervernieuwing », Dramatisch akkoord, Amsterdam, Van Kampen, p. 47-50.

Tindemans, Carlos, 1969, tapuscript, le 5 avril 1969 (publié dans Kunst \& Cultuur, le 16 avril 1969). Collection d'archives et de documentation Carlos Tindemans, Brussel, Vlaams Theaterinstituut.

Tindemans, Carlos (dir.), 1989, Op de top van de citadel : over Alex Van Royen, Antwerpen, Dedalus. 


\section{NOTES}

1. Sur la personne d'Alex Van Royen voir Tindemans (1989).

2. Cette citation et les suivantes sont tirées du manifeste: Hugo Claus, Carlos Tindemans et Alex Van Royen, 1968, T68 of de toekomst van het theater in Zuid-Nederland, Edegem-Antwerp, n. p. (ma traduction).

3. C'est précisément pour cette raison que leur manifeste ne trouvera pas d'échos dans les milieux progressistes dont certains courants considéraient le théatre comme un outil politique ou même révolutionnaire.

4. Ce faisant, les auteurs semblent se distancier de manière implicite de compagnies telles que le Living Theatre, Bread and Puppet Theatre et la Performance Group, caractérisées par la coïncidence du processus de recherche et du résultat final.

5. La discussion relative à la formation de l'acteur est liée à l'arrêté royal du 18 février 1964, réalisé grâce à l'influence du fonctionnaire culturel Alfons Van Impe, maudit par Tindemans. Cet AR prévoit que la distinction entre les acteurs professionnels et les amateurs repose sur la possession ou non d'un diplôme de conservatoire. Une disposition qui serait source de polémique : «à cette époque, l'on aimait se prévaloir de ce soi-disant don général, spontané que possèderaient les Flamands pour le théâtre " (Thielemans $2007: 89$ ). Et ce don, d'après cette hypothèse, pourrait se passer d'une formation. Les théâtres officiels devaient compter au moins douze acteurs « professionnels » dans leur compagnie.

6. Carlos Tindemans, tapuscript, le 5 avril 1969 (plus tard repris dans Kunst \& Cultuur, le 16 avril 1969). Collection d'archives et de documentation Carlos Tindemans, Vlaams Theaterinstituut Brussel.

7. De Nieuwe Gazet, le 24 mai 1962.

8. Voir aussi Decreus (1996).

\section{RÉSUMÉS}

En 1968, un trio d'artistes composé de l'écrivain Hugo Claus, du comédien Alex Van Royen et du critique Carlos Tindemans, lance son manifeste intitulé T68, que Claus fera rapidement publier et distribuer en deux cents copies. Dans ce manifeste les trois protagonistes, en s'attaquant à ce qu'ils appellent eux-mêmes la sclérose du théâtre bourgeois, défendent l'autonomie fondamentale d'un théâtre libéré de contraintes politiques, institutionnelles et morales et revendiquent la nécessité d'un renouveau théâtral en Flandre dont ils espèrent qu'il se réalisera grâce à la fondation d'un nouveau théâtre dit «national» accompagné d'un laboratoire expérimental. La présente contribution défend l'hypothèse que ce "manifeste malgré lui » occupe une position unique mais paradoxale au sein de l'historiographie récente du théâtre d'expression néerlandophone en Belgique. À la fois révolutionnaire et réactionnaire, ce texte, qui proclame la nécessité d'une révolution profonde, n'a pas pu se connecter aux mouvements antiautoritaires de l'époque et se trouvera donc vite réduit à un « curiosum ».

In 1968, a trio of artists composed of writer Hugo Claus, actor Alex Van Royen and critique Carlos Tindemans, launched their manifesto entitled T68, which Claus will have published and distributed in two hundred copies shortly after. In this manifesto, the three men firmly criticize what they describe as the sclerosis of bourgeois theatre, while at the same time defending the 
fundamental autonomy of a theatre liberated of all possible political, institutional and moral constraints. They demand a necessary and fundamental renewal of Flemish theatre life putting all their hopes on foundation of a new, so-called national theatre that should equally entail a laboratory for experimental research. This paper defends the hypothesis that this manifesto "in spite of itself" occupies a unique but paradoxical position within the recent historiography of Dutch-speaking theatre in Belgium. Both revolutionary and profoundly conservative, this text seemed out of joint with the then anti-authoritarian currents and saw itself (too?) quickly reduced to the status of historical curiosity.

\section{INDEX}

Mots-clés : manifeste, théâtre flamand, renouvellement théâtral, mai 1968, théâtre

Keywords : manifesto, theatre, Flemish theatre, May 1968, theatrical renewal

\section{AUTEUR}

\section{KAREL VANHAESBROUCK}

Université libre de Bruxelles, CiASp (Centre de recherche en cinéma et arts du spectacle) 